\title{
All-optical pulse-train generation and distributed sensing through the temporal analogue of a laser
}

Alexis Cristian Sparapani ( $\square$ alexis.sparapani@ib.edu.ar)

Centro Atomico Bariloche https://orcid.org/0000-0002-0139-1811

\section{Germán Fernández}

Centro Atomico Bariloche

Alfredo Sánchez

Centro Atomico Bariloche

Juan Bonetti

Centro Atomico Bariloche

Nicolás Linale

Centro Atomico Bariloche

Diego Grosz

Centro Atomico Bariloche

\section{Research Article}

Keywords: Nonlinear Optics, Temporal Laser, Nonlinear Fibers, Distributed Sensing

Posted Date: March 30th, 2021

DOI: https://doi.org/10.21203/rs.3.rs-321469/v1

License: (c) (i) This work is licensed under a Creative Commons Attribution 4.0 International License.

Read Full License 


\title{
All-optical pulse-train generation and distributed sensing through the temporal analogue of a laser
}

\author{
A. Sparapani ${ }^{*(a)(b)}$. G. Fernández ${ }^{(b)}$. \\ A. D. Sánchez ${ }^{(a)(b)}$. J. Bonetti ${ }^{(a)(b)}$. \\ N. Linale ${ }^{(a)(b)}$. D. F. Grosz ${ }^{(a)(b)}$
}

Received: date / Accepted: date

\begin{abstract}
We propose a novel scheme for the all-optical generation of pulse trains in optical fibers based on the reflection and refraction of pulses at a time boundary, and the temporal analogue of a laser cavity with gain. Furthermore, this scheme is shown to provide an original and simple way to measure distributed fiber parameters. In particular, we put forth an application of the temporal laser to the distributed sensing of the fiber group-velocity dispersion parameter.
\end{abstract}

Keywords Nonlinear Optics · Temporal Laser · Nonlinear Fibers · Distributed Sensing

\section{Introduction}

Optical fibers exhibit multiple effects that modify the characteristics of propagating pulses. The dependence of the refractive index on frequency gives rise to chromatic dispersion and the wavelength dependence of the group velocity; fiber attenuation results in the loss of the pulse energy [1]. In addition to these linear effects, nonlinear effects have been shown to significantly restrict the Shannon capacity of optical fibers [2].

In particular, the optical Kerr effect, i.e, the dependence of the refractive index on intensity $[1,3,4]$ has been widely studied to prevent its detrimental action in optical communication systems $[5,6]$, but also lends itself to a vast number of applications in photonics, such as in frequency conversion $[7,8]$ and supercontinuum generation $[9,10]$, to name a few.

One interesting application of the fiber nonlinearity lies on performing analogies with other diverse phenomena whose direct experimental observation might be difficult to perform. Relevant examples can be found, for instance, in the fiberoptical analogue of event horizons and black holes [11,12], in the analogy between electrons and photons in quantum tunneling [13], and in the study of optical rogue

*email: alexis.sparapani@ib.edu.ar

${ }^{(a)}$ Consejo Nacional de Investigaciones Científicas y Técnicas (CONICET), Argentina.

${ }^{(b)}$ Depto. de Ingeniería en Telecomunicaciones, GDTyPE, GAIyANN, Centro Atómico Bariloche, Comisión Nacional de Energía Atómica, Río Negro 8400, Argentina. 
waves [14], an analogy with an extreme phenomenon widely studied in hydrodynamics, among others.

In the past years, the temporal analogue of reflection and refraction laws has attracted the attention of the nonlinear optics community [15-17]. It relies on the Kerr effect induced by high-intensity pulses propagating in a nonlinear fiber that play the role of moving refractive-index boundaries [18, 19]. As explained in the next section, an intense pulse is found to produce a time-dependent refractive index for another co-propagating low-intensity pulse with a different group velocity. This basic idea can be used, for instance, to perform the temporal analogy of a boundary separating two media with different refractive indices. A low-intensity pulse reaching that boundary is split into two pulses with different group velocities, resembling the behavior of reflected and refracted pulses. As shown in Ref. [16], the frequency shift involved in the change of group velocities completes this analogy by acting as the incident and refracted angles in Snell's law. This scheme has been exploited to study different applications such as the splitting of pulses [20], wavelength conversion, optical delays, and pulse manipulation in the time and frequency domains [17], the study of plasmonic behavior [21], pulse interference [20], and many others $[18,19,22,23]$.

In this work, we propose to extend this analogy of reflection and refraction to devise a novel scheme of a temporal analogue of a laser. This proposal, portrayed in Fig 1, relies on a temporal Fabry-Pérot cavity shaped by the Kerr-induced refractive index change, $\Delta n$, prompted by two different high-intensity pulses. Each pulse acts as a potential barrier for a third low-intensity pulse confined within the temporal cavity which is periodically reflected at the time boundaries. The transmission coefficient of each barrier depends on the intensity and duration, $\Delta T$, of the high-intensity pulse. In this particular configuration, one barrier is set to satisfy the total internal reflection (TIR) condition for the low-intensity pulse, while the second barrier has a nonzero transmission coefficient, leading to the generation of an output train of pulses with a periodicity related to the cavity length, $T_{\text {cav }}$. In order to compensate the energy loss due to the nonzero transmission in the second barrier, we propose to provide the temporal cavity with a gain mechanism resembling the active medium in a laser. By proper adjustment of this gain, the transmission loss can be exactly compensated and thus a constant-amplitude output train of pulses can be achieved. Further, we show this scheme to easily lend itself to the distributed sensing of fiber and pulse parameters. In particular, we put forth an example of the application of the temporal cavity to the distributed measurement of the fiber group-velocity dispersion (GVD) parameter [24-26]. Having accurate data of the GVD parameter along an optical fiber link is critical for the design of efficient dispersion maps in long-haul networks, for which dispersion monitoring and compensation is indispensable in order to improve transmission performance [27-30]. In addition, the knowledge of the spatial variation of the GVD parameter in fibers is of singular relevance when considering the transmission of dispersion-managed solitons in ultra-long-haul fiber-optic communication systems [31-33].

The paper is organized as follows: Section 2 introduces the temporal laser model. Section 3 shows numerical results on the implementation of the proposed scheme, and Section 4 discusses the application to the distributed sensing of the GVD parameter. Finally, in Section 5 we present our conclusions. 


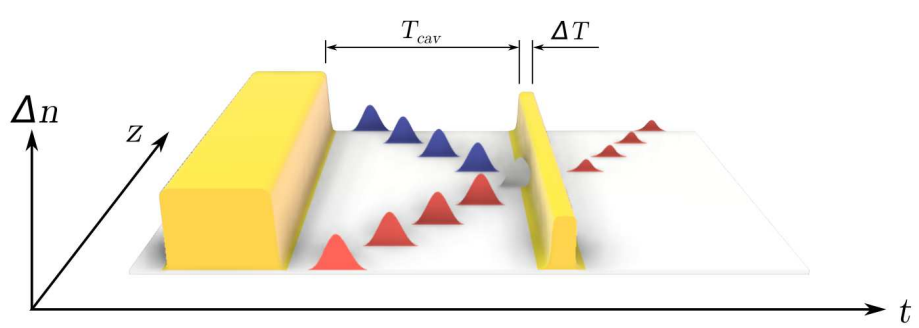

Fig. 1: Schematic diagram of the temporal cavity. The thick barrier acts as a perfect mirror, while the narrow barrier, of width $\Delta T$, acts as a highly reflective output mirror. $T_{\text {cav }}$ is the temporal analogue of the cavity length, and $\Delta n$ stands for the refractive index modulation produced by the high-intensity pulses

\section{Model description}

The temporal analogy of reflection and refraction has already been demonstrated in media with a time-dependent refractive indices $[15-17,34]$. As it was mentioned in the previous section, this can be achieved by means of an intense ( $p u m p)$ pulse propagating in an optical fiber, where the change in the refraction index is a consequence of the optical Kerr effect. The propagation of a low-amplitude (signal) pulse in a pump co-moving frame is modeled by the equation $[16,34]$

$$
\frac{\partial A}{\partial z}+\Delta \beta_{1} \frac{\partial A}{\partial t}+i \frac{\beta_{2}}{2} \frac{\partial^{2} A}{\partial t^{2}}=i \gamma P(t) A
$$

where $z$ is the propagation axis, $A$ is the complex envelope of the signal, $P(t)$ is the pump power (assumed to be constant along $z$ ), $\gamma$ is the fiber nonlinear coefficient, $\Delta \beta_{1}$ is the group-velocity mismatch between pump and signal, and $\beta_{2}$ is the GVD parameter. An enlightening case of Eq. 1 is given by $P(t)=P_{0} H\left(t-T_{\mathrm{B}}\right)$, where $P_{0}$ is a constant power, $H$ stands for the Heaviside function and $T_{\mathrm{B}}$ is the temporal analogue of a boundary separating two media with different refractive indices. A complete analysis of the analogy of reflected and refracted pulses and the TIR condition can be found in Ref. [16]. However, the analogy provided by the more general expression in (Eq. 1) and its potential applications has not yet been exploited to its fullest.

In this work we propose a different pumping scheme with

$$
P(t)=P_{1} H\left(-\frac{2 t}{T_{\mathrm{cav}}}-\frac{1}{2}\right)+P_{2} \Pi\left(\frac{t-T_{\mathrm{cav}} / 2}{\Delta T}-\frac{1}{2}\right)
$$

where $\Pi$ is the rectangular function. This modulation of the pump power generates a temporal analogue of a Fabry-Pérot cavity, which we shall henceforth call a temporal cavity, as shown schematically in Fig. 1. The Heaviside function effectively models the wide pulse leading to a change in the refractive index, weighted by $P_{1}$ and adjusted to produce the TIR of the signal. On the other hand, the rectangular function of width $\Delta T$ acts as a potential barrier, whose transmission coefficient $T_{r}$ depends on $P_{2}$ and $\Delta T$, and can be calculated analytically by the standard 

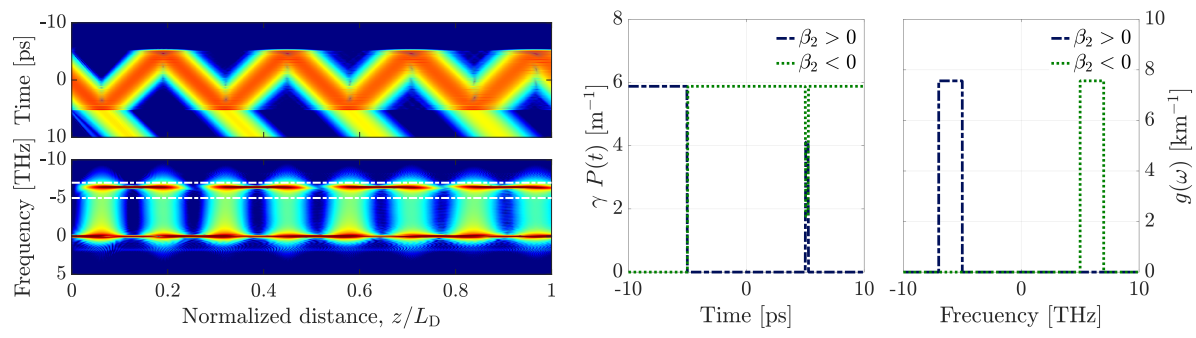

Fig. 2: (Left) Simulation of the temporal laser in the time and frequency domains for $\beta_{2}>0$. Each reflection/refraction produces a frequency shift corresponding to the change in the group velocity of the signal pulse. Pump configuration (center) and the frequency-dependent gain for normal and anomalous dispersion regimes (right)

analysis of a one-dimensional electromagnetic cavity [35] leading to

$$
T_{r}=\frac{1}{\left|\Gamma^{+}+\Gamma^{-}\right|^{2}},
$$

with $\Gamma^{ \pm}=\omega^{ \pm} \exp \left(i \omega^{ \pm} \Delta T\right)\left(1+\omega^{\mp} / \omega_{r}\right) /\left(\omega^{ \pm}-\omega^{\mp}\right)$, where the frequencies are defined as $\omega^{ \pm}=\left(-\Delta \beta_{1} \pm \sqrt{\left(\Delta \beta_{1}\right)^{2}-2\left|\beta_{2}\right| \gamma P_{2}}\right) / \beta_{2}$, and $\omega_{r}=-2 \Delta \beta_{1} / \beta_{2}$. If $0<T_{r}<1$ successive transmitted pulses conform a pulse train of period $2 T_{\text {cav }}$. However, the amplitude of these pulses is expected to decrease, as the energy of the original signal pulse is sequentially split at the barrier. In order to obtain a constant-amplitude output pulse train we require the analogue of an active medium within the cavity, thus completing the analogy of a temporal laser. The optical gain can be included in the propagation equation as

$$
\frac{\partial A}{\partial z}+\Delta \beta_{1} \frac{\partial A}{\partial t}+i \frac{\beta_{2}}{2} \frac{\partial^{2} A}{\partial t^{2}}-\frac{\hat{g}}{2} A=i \gamma P(t) A,
$$

where $\hat{g}$ is the frequency-dependent gain operator, defined as $\hat{g} A=\mathcal{F}^{-1}[g(\omega) \mathcal{F}(A)]$, where $g(\omega)$ is the gain profile and $\mathcal{F}$ stands for the Fourier transform. In a practical implementation this gain can be realized, for instance, by an external laser providing Raman gain in the transmission fiber [1]. Furthermore, this gain is required to have a limited bandwidth $\Delta \omega$ around the frequency of the reflected pulse, $\omega_{r}$, as it is explained below. In this way, we can easily estimate the gain required to compensate for transmission losses, allowing for the generation of a constant-amplitude output pulse train, by proposing $g(\omega)=g_{\mathrm{eq}} \Pi\left(\left(\omega-\omega_{r}\right) / \Delta \omega\right)$, where

$$
g_{\text {eq }}=\frac{\Delta \beta_{1}}{T_{\text {cav }}} \ln \left(\frac{1}{1-T_{r}}\right) \text {. }
$$

Note that the scheme discussed so far is strictly valid for positive values of the GVD parameter, i.e., the normal dispersion regime. The temporal laser can also be achieved in the anomalous dispersion regime by replacing the pump configuration of Eq. 2 by

$$
P(t)=P_{1}-\left[P_{1} H\left(-\frac{2 t}{T_{\mathrm{cav}}}-\frac{1}{2}\right)+P_{2} \Pi\left(\frac{t-T_{\mathrm{cav}} / 2}{\Delta T}-\frac{1}{2}\right)\right],
$$

as suggested in Ref. [34]. 


\section{Numerical simulations and validation of the model}

In order to observe the dynamics of the proposed model, we perform an illustrating numerical simulation of Eq. 4 by resorting to the Split-Step Fourier, an algorithm widely used in the realm of nonlinear fiber optics [1]. Parameters are set to $\Delta \beta_{1}=$ $0.42 \mathrm{ps} / \mathrm{km}, \beta_{2}= \pm 21 \mathrm{ps}^{2} / \mathrm{km}, T_{\text {cav }}=10 \mathrm{ps}, \Delta T=0.25 \mathrm{ps}, \gamma P_{1}=5.88 \mathrm{~m}^{-1}$, $\gamma P_{2}=4.12 \mathrm{~m}^{-1}$, and the fiber length is $L_{\mathrm{D}}$, where $L_{\mathrm{D}}=T_{0}^{2} /\left|\beta_{2}\right|$ is the dispersion length. The input signal is a $T_{0}=2$-ps $0.1-\mathrm{W}$ Gaussian pulse centered at $\lambda=$ $1550 \mathrm{~nm}$. Figure 2 (left panel) shows simulation results in the time and frequency domains in the normal dispersion regime. Observe how the evolution of the signal pulse along propagation mimics the temporal dynamics of a pulse confined within a Fabry-Pérot cavity. In addition, the nonzero transmission coefficient of the right barrier allows for the periodical emission of transmitted pulses from the temporal cavity, in direct analogy with the output mirror of a laser cavity.

Note that since the propagation distance is commensurate with the fiber characteristic dispersion length, the temporal broadening of the output pulses is not appreciable in Fig. 2. Further, fiber dispersion does not affect the regularity of the generated pulse train, as each output pulse from the cavity experiences the same distortion, and pulse broadening depends on the total propagated distance by the pulse both within and outside the temporal cavity.

The spectrum of signal pulses is also shown in Fig. 2 (left), showcasing the discrete frequency shift associated with the temporal reflection at each time boundary, in agreement with results in Ref. [20]. This singular feature associated with the reflection at a time boundary suggests a way to design an adequate frequencydependent gain, based on the direction-dependent amplification of the signal pulse, in this case when the signal pulse is traveling towards the thick barrier. This original scheme avoids the overlapping of the gain and the transmission in the right barrier (output), allowing for the simple estimation of $T_{r}$ given by Eq. 3. Figure 2 (center panel) shows the different pump configurations required for the two dispersion regimes, according to Eq. 2 for normal dispersion, and Eq. 6 for anomalous dispersion, and Fig. 2 (right) shows the corresponding gain frequency band.

Figure 3 displays a comparison between the pulse train at the output end of the fiber as obtained with and without gain in the temporal cavity, where the amplitude is normalized to that of the input Gaussian pulse. In the absence of gain in the temporal cavity, the pulse train displays an expected progressive decrease of energy due to the periodical splitting at the output barrier. It must be noted that pulses on the right were emitted at an earlier propagation stage than the pulses on the left and, as a consequence, they exhibit a larger peak power. On the other hand, the pulse train obtained with the narrowband gain $g_{\text {eq }}$ in the temporal cavity displays a notable periodicity and constant energy per pulse, validating the estimation made with Eq. 5.

\section{Distributed sensing of fiber parameters}

The temporal analogue of a laser proposed in this paper can be regarded as a moving laser cavity propagating in the fiber. From this point of view, the output pulse train consists of a sequence of pulses generated at different points, $z_{n}$, equally distributed along the fiber. As a consequence, each pulse is expected to encase 


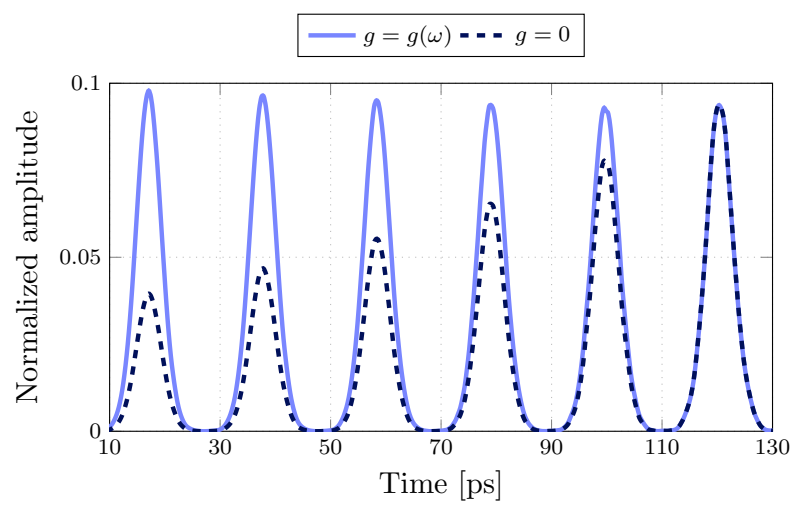

Fig. 3: Pulse train generated by the temporal cavity (dashed) and the temporal laser (solid), obtained at the fiber output. Simulation parameters are the same as those in Fig. 2 but for a propagation distance of $1.5 L_{\mathrm{D}}$

certain information about the parameters of the fiber at $z_{n}$, i.e., the point at which it exited the cavity. This suggests the potential application of the temporal laser as a scheme capable of performing the distributed sensing of fiber parameters. As an example, in this section we introduce a simple method to measure variations in the GVD parameter along the fiber with respect to a nominal value.

Figure 4 (top panel) shows the output pulse train for a fiber with a nominal GVD parameter of $\beta_{2}=21 \mathrm{ps}^{2} / \mathrm{km}$. In this case, the gain estimated with Eq. 5 provides a balance between amplification and losses by transmission, producing an output pulse train of constant amplitude. In the same figure we show the output pulse train generated by the same temporal-laser configuration, this time in a fiber with a slightly different $\beta_{2}$. We observe that a small change in the GVD parameter leads to a change in the transmission coefficient; consequently, the balance between gain and loss is no longer maintained as $g_{\mathrm{eq}}$ was set for the nominal value of $\beta_{2}$. For instance, the pulse train obtained in a fiber with a lower GVD parameter $\left(\beta_{2}=20.5 \mathrm{ps}^{2} / \mathrm{km}\right)$ shows a progressive increase of the pulse energy, evidencing an overcompensation of transmission losses. On the other hand, for a higher GVD parameter $\left(\beta_{2}=21.5 \mathrm{ps}^{2} / \mathrm{km}\right)$ transmission losses are not compensated by $g_{\text {eq }}$, producing a successive decrease of the pulse energy in the output train. This way, the increase/decrease of the pulse energy, which can be easily measured, can be used to detect deviations in the GVD parameter along the fiber. Figure 4 (bottom panel) shows that the energy ratio between two successive pulses can be univocally associated to a value of $\beta_{2}$, for every $E_{n+1} / E_{n}$, thus providing a simple transduction scheme.

Although results shown in Fig. 4 (top panel) correspond to simulations of a fiber with fixed values of $\beta_{2}$, this scheme can be applied to the measurement of a $z$-dependent GVD parameter. The value of $\beta_{2}$ at the point $\left(z_{n}+z_{n-1}\right) / 2$ can be inferred by computing the ratio between the energy of consecutive pulses in the output train $E_{n} / E_{n-1}$, being $E_{n}$ the energy of the pulse generated at $z_{n}$, and using the calibration from Fig. 4 (bottom panel). Following this method, each pulse train can be converted into a discrete $z$-mapping of the fiber GVD parameter, whose resolution in $z$ is given by $2 T_{\text {cav }} / \Delta \beta_{1}$, and can thus be designed 
to meet the desired resolution. Note also that since the measured magnitude in this transduction scheme is the pulse energy, detection can be conveniently performed with a photodetector of small bandwidth.

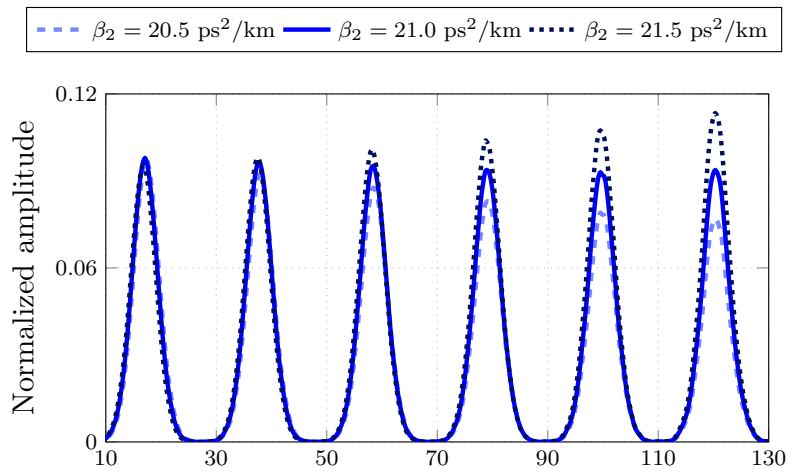

Time $[\mathrm{ps}]$

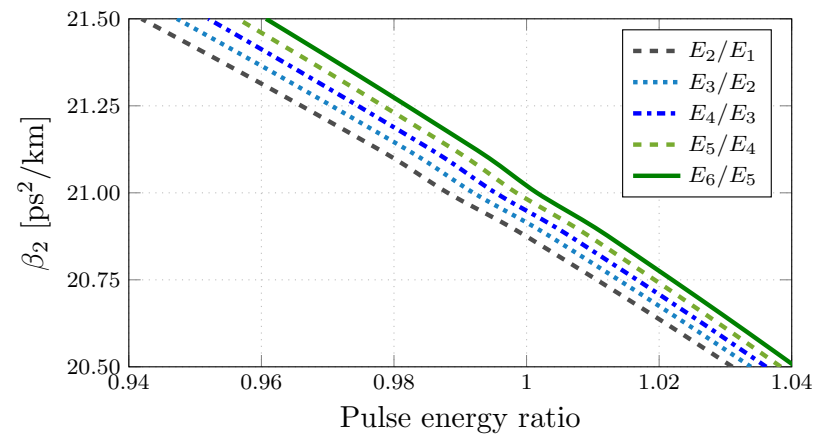

Fig. 4: (Top) Temporal laser output for different values of $\beta_{2}$. A constant-amplitude pulse train is obtained only for the nominal value of $\beta_{2}$. (Bottom) Energy ratio between consecutive output pulses vs. $\beta_{2}$. $E_{n}$ stands for the energy of the $n$-th output pulse

\section{Conclusions}

In this work we presented a temporal laser model departing from the temporal analogy of reflection and refraction laws. This proposal consists of a temporal cavity scheme plus a frequency-selective gain playing the role of the active medium in a conventional laser. The output pulse train obtained with the temporal laser was also shown to find applications in the distributed sensing of fiber parameters. As an enlightening example, we showed the distributed sensing of variations of the fiber GVD parameter with respect to a nominal value by the direct and simple measurement of the energy ratio of successive output pulses. 


\section{Declarations}

Not applicable.

\section{References}

1. G.P. Agrawal, Nonlinear Fiber Optics, 6th ed. (Academic Press, 2019)

2. R.J. Essiambre, G. Kramer, P.J. Winzer, G.J. Foschini, B. Goebel, Journal of Lightwave Technology 28(4), 662 (2010)

3. R.W. Boyd, Nonlinear Optics, 3rd ed. (Academic Press, 2008)

4. P.E. Powers, J.W. Haus, Fundamentals of Nonlinear Optics, 2nd ed. (CRC press, 2017)

5. F. Forghieri, R.W. Tkach, A.R. Chraplyvy, D. Marcuse, IEEE Photonics Technology Letters 6(6), 754 (1994)

6. M.W. Maeda, W.B. Sessa, W.I. Way, A. Yi-Yan, L. Curtis, R. Spicer, R.I. Laming, Journal of Lightwave Technology 8(9), 1402 (1990)

7. A.V. Gorbach, D.V. Skryabin, Physical Review A 76(5), 053803 (2007)

8. R. Dekker, A. Driessen, T. Wahlbrink, C. Moormann, J. Niehusmann, M. Först, Optics Express 14(18), 8336 (2006)

9. R. Driben, A.V. Yulin, A. Efimov, B.A. Malomed, Optics Express 21(16), 19091 (2013)

10. J.M. Dudley, G. Genty, S. Coen, Reviews of Modern Physics 78(4), 1135 (2006)

11. T.G. Philbin, C. Kuklewicz, S. Robertson, S. Hill, F. König, U. Leonhardt, Science 319(5868), 1367 (2008)

12. S. Robertson, U. Leonhardt, Physical Review A 81(6), 063835 (2010)

13. R.Y. Chiao, P.G. Kwiat, A.M. Steinberg, Physica B 175(1-3), 257 (1991)

14. D.R. Solli, C. Ropers, P. Koonath, B. Jalali, Nature 450(7172), 1054 (2007)

15. J.T. Mendonça, P.K. Shukla, Physica Scripta 65(2), 160 (2002)

16. B.W. Plansinis, W.R. Donaldson, G.P. Agrawal, Physical Review Letters 115(18), 183901 (2015)

17. M.A. Gaafar, T. Baba, M. Eich, A. Petrov, Nature Photonics 13(11), 737 (2019)

18. V.E. Lobanov, A.P. Sukhorukov, Physical Review A 82(3), 033809 (2010)

19. K.E. Webb, M. Erkintalo, Y. Xu, N.G.R. Broderick, J.M. Dudley, G. Genty, S.G. Murdoch, Nature Communications 5(1), 1 (2014)

20. B.W. Plansinis, W.R. Donaldson, G.P. Agrawal, Journal of the Optical Society of America B 34(10), 2274 (2017)

21. G.A. Menendez, B. Maes, Optics Letters 42(23), 5006 (2017)

22. M.A. Gaafar, D. Jalas, L. O'Faolain, J. Li, T.F. Krauss, A.Y. Petrov, M. Eich, Nature Communications 9(1), 1 (2018)

23. M.A. Gaafar, H. Renner, A.Y. Petrov, M. Eich, Optics Express 27(15), 21273 (2019)

24. M. González-Herráez, L. Thévenaz, IEEE Photonics Technology Letters 16(4), 1128 (2004)

25. L.F. Mollenauer, P.V. Mamyshev, J. Gripp, M.J. Neubelt, N. Mamysheva, L. GrünerNielsen, T. Veng, Optics Letters 25(10), 704 (2000)

26. M. González-Herráez, L. Thévenaz, P. Robert, Optics Letters 28(22), 2210 (2003)

27. C. Furst, C. Scheerer, G. Mohs, J.P. Elbers, C. Glingener, in OFC 2001. Optical Fiber Communication Conference and Exhibit. Technical Digest Postconference Edition (IEEE Cat. 01CH37171), vol. 1 (IEEE, 2001), vol. 1, pp. MF4-MF4

28. K. Forozesh, S.L. Jansen, S. Randel, I. Morita, H. Tanaka, in 2008 Digest of the IEEE/LEOS Summer Topical Meetings (IEEE, 2008), pp. 135-136

29. M.Y. Hamza, I. Hayee, in 2016 IEEE 10th International Conference on Application of Information and Communication Technologies (AICT) (IEEE, 2016), pp. 1-5

30. J. Lee, Current Optics and Photonics 3(3), 193 (2019)

31. U. Teğin, E. Kakkava, B. Rahmani, D. Psaltis, C. Moser, in 2020 Conference on Lasers and Electro-Optics (CLEO) (IEEE, 2020), pp. 1-2

32. T. Yu, E.A. Golovchenko, A.N. Pilipetskii, C.R. Menyuk, Optics Letters 22(11), 793 (1997)

33. A.P. Küng, A. Agarwal, D.F. Grosz, S. Banerjee, D.N. Maywar, Journal of Lightwave Technology 23(3), 1182 (2005)

34. J. Zhou, G. Zheng, J. Wu, Physical Review A 93(6), 063847 (2016)

35. J.D. Jackson, Classical Electrodynamics, 3rd ed. (John Wiley \& sons, 1999) 


\section{Figures}

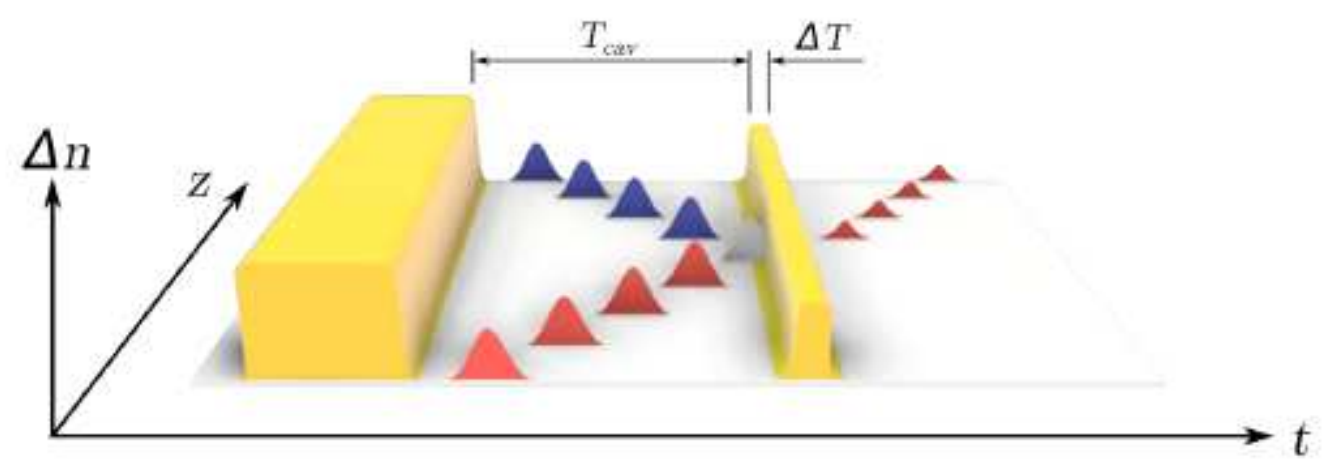

\section{Figure 1}

Schematic diagram of the temporal cavity. The thick barrier acts as a perfect mirror, while the narrow barrier, of width $\Delta T$, acts as a highly re ective output mirror. Tcav is the temporal analogue of the cavity length, and $\Delta \mathrm{n}$ stands for the refractive index modulation produced by the high-intensity pulses
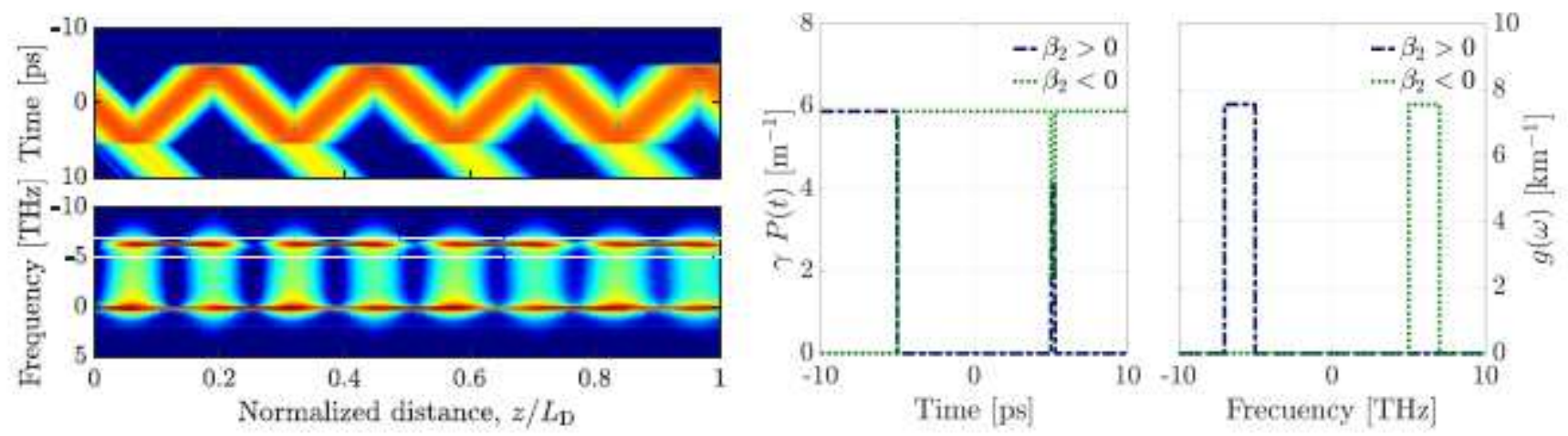

Figure 2

(Left) Simulation of the temporal laser in the time and frequency domains for $\beta 2>0$. Each reflection/refraction produces a frequency shift corresponding to the change in the group velocity of the signal pulse. Pump configuration (center) and the frequency-dependent gain for normal and anomalous dispersion regimes (right) 


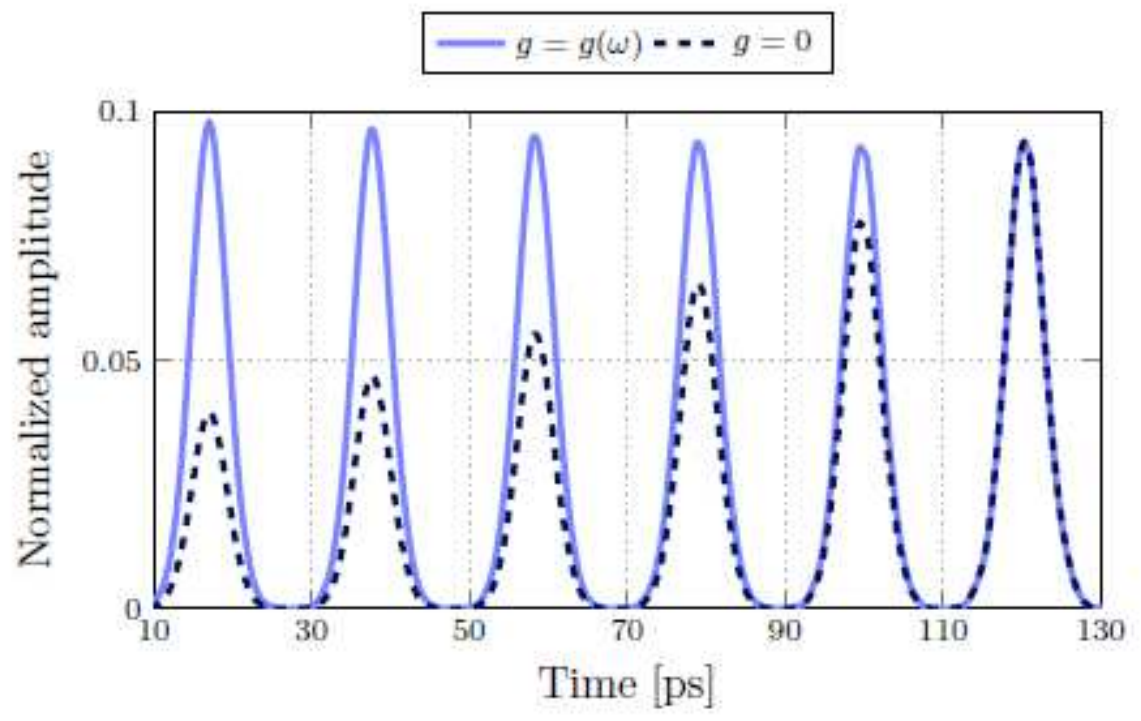

Figure 3

Pulse train generated by the temporal cavity (dashed) and the temporal laser (solid), obtained at the ber output. Simulation parameters are the same as those in Fig. 2 but for a propagation distance of 1:5LD
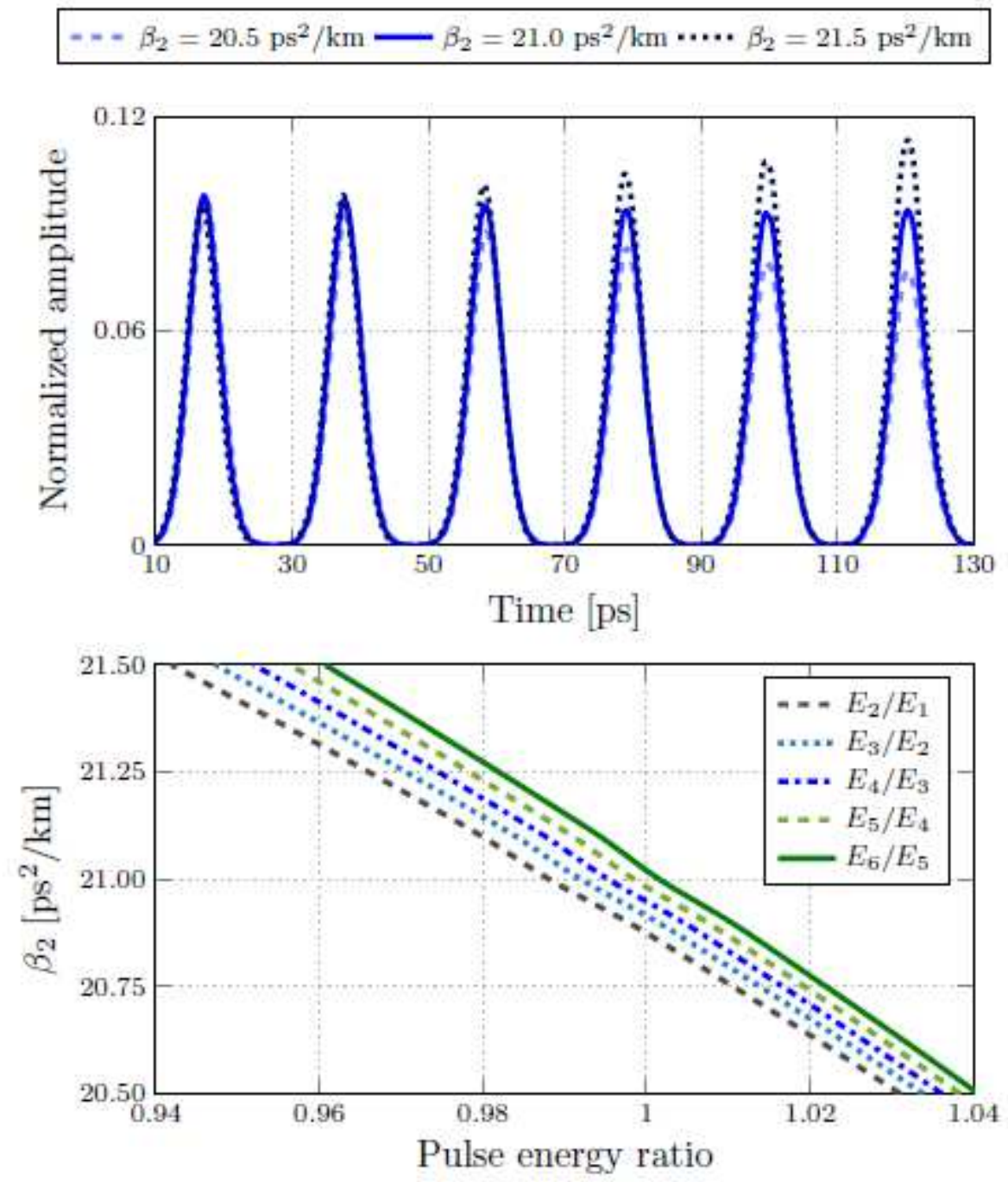
Figure 4

(Top) Temporal laser output for different values of $\beta 2$. A constant-amplitude pulse train is obtained only for the nominal value of $\beta 2$. (Bottom) Energy ratio between consecutive output pulses vs. $\beta 2$. En stands for the energy of the $n$-th output pulse 